\title{
COINCIDENCE THEOREMS IN COMPLETE METRIC SPACES
}

\author{
Y. J. CHO, N. J. HUANG AND L. XIANG
}

\begin{abstract}
The purpose of this paper is to introduce new classes of generalized contractive type set-valued mappings and weakly dissipative mappings and to prove some coincidence theorems for these mappings by using the concept of $\omega$-distances.
\end{abstract}

\section{Introduction}

Recently, in [6] and [8], Kada-Suzuki-Takahashi introduced the new concept of $\omega$ distances in metric spaces and improved Caristi's fixed point theorem, Ekeland's $\varepsilon$ variational principle and the nonconvex minimization theorem in metric spaces.

On the other hand, in [5], Husain-Latif introduced a class of generalized contractive type set-valued mappings in metric spaces and showed the existence of fixed points for these mappings ([3], [4], [9]).

In this paper, we introduce new classes of generalized contractive type set-valued mappings and weakly dissipative mappings in metric spaces and prove some coincidence theorems for these mappings by using the concept of $\omega$-distances. Our main results extend, generalize and improve the results of Caristi ([1]), Nadler ([17]), Kada-SuzukiTakahashi ([6]) and others ([2]-[5], [9]).

\section{Preliminaries}

Throughout this paper, let $N$ and $R$ denote the sets of positive integers and real numbers, respectively.

Definition 2.1. ([6]) Let $(X, d)$ be a metric space. A function $p: X \times X \rightarrow[0, \infty)$ is called a $\omega$-distance on $X$ if the following conditions are satisfied:

(1) $p(x, z) \leq p(x, y)+p(y, z)$ for all $x, y, z \in X$,

(2) for any $x \in X, p(x, \cdot): X \rightarrow[0, \infty)$ is lower semicontinuous,

Received April 19, 1996.

1991 Mathematics Subject Classification. 47H10, 54H25, 54C60

Key words and phrases. $\omega$-distance, coincidence point, contractive type set-valued mapping, weakly dissipative mapping.

The first author was supported in part by the Academic Research Fund of Ministry of Education, Korea, 1997, Project No. BSRI-97-1405. 
(3) for any $\varepsilon>0$, there exists a number $\delta>0$ such that $p(z, x) \leq \delta$ and $p(z, y) \leq \delta$ imple $d(x, y) \leq \varepsilon$.

Some examples of $\omega$-distances are given as follows:

Example. ([6]) (1) If $X$ is a metric space with the metric $d$, then $p=d$ is a $\omega$-distance on $X$.

(2) If $X$ is a normed linear space with the norm $\|\cdot\|$, then a function $p: X \times X \rightarrow[0, \infty)$ defined by $p(x, y)=\|x\|+\|y\|$ for all $x, y \in X$ is a $\omega$-distance on $X$.

(3) Let $(X, d)$ be a metric space. If $T$ is a continuous mapping from $X$ into itself, then a function $p: X \times X \rightarrow[0, \infty)$ defined by $p(x, y)=\max \{d(T x, y), D(T x, T y)\}$ for all, $x, y \in X$ is a $\omega$-distance on $X$.

(4) Let $(X, d)$ be a metric space and $F$ be a bounded and closed subset of $X$. If $F$ contains at least two points and $C$ is a constant with $C \geq \operatorname{diam} F$, where diam $F$ denotes the diameter of $F$, then a function $p: X \times X \rightarrow[0, \infty)$ defined by

$$
p(x, y)= \begin{cases}d(x, y) & \text { for } x, y \in F \\ C & \text { for } x \notin F \text { or } y \notin F\end{cases}
$$

is a $\omega$-distance on ${ }^{\prime} X$.

We need the following lemma for our main theorems:

Lemma 2.1. ([6]) Let $(X, d)$ be a metric space, $p$ be a $\omega$-distance and $\left\{x_{n}\right\},\left\{y_{n}\right\}$ be sequences in $X$. Let $\left\{a_{n}\right\}$ and $\left\{\beta_{n}\right\}$ be sequences in $[0, \infty)$ converging to 0 and let $x, y, z \in X$. Then we have the following:

(1) If $p\left(x_{n}, y\right) \leq a_{n}$ and $p\left(x_{n}, z\right) \leq \beta_{n}$ for any $n \in N$, then $y=z$. In particular, if $p(x, y)=0$ and $p(x, z)=0$, then $y=z$.

(2) If $p\left(x_{n}, y_{n}\right) \leq a_{n}$ and $p\left(x_{n}, z\right) \leq \beta_{n}$ for any $n \in N$, then $\left\{y_{n}\right\}$ converges to $z$.

(3) If $p\left(x_{n}, x_{m}\right) \leq a_{n}$ for any, $n, m \in N$ with $m>n$, then $\left\{x_{n}\right\}$ is a Cauchy sequence in $X$.

(4) If $p\left(y, x_{n}\right) \leq a_{n}$ for any $n \in N$, then $\left\{x_{n}\right\}$ is a Cauchy sequence in $X$.

Let $2^{X}$ denote the family of all nonempty subsets of a metric space $(X, d)$.

Definition 2.2. Let $(X, d)$ be a metric space and $M$ be a nonempty subset of $X$. Let $p$ be a $\omega$-distance on $X$ and $f$ be a function from $M$ into $X . J: M \rightarrow 2^{X}$ is said to be a weakly $f$-contractive type set-valued mapping if, for all $x \in M$ and $u_{x} \in J x$, there exists $v_{y} \in J y$ for all $y \in M$ such that

$$
p\left(u_{x}, v_{y}\right) \leq h p(f x, f y)
$$

for some $h \in[0,1)$. 
Defimition 2.3. ([16]) Let $(X, d)$ be a metric space and $p$ be a $\omega$-distance on $X$. A set-valued mapping $J: X \rightarrow 2^{X}$ is said to be weakly contractive (or p-contractive) if, for any $x_{1}, x_{2} \in X$ and $y_{1} \in J x_{1}$, there exists $y_{2} \in J x_{2}$ such that

$$
p\left(y_{1}, y_{2}\right) \leq r p\left(x_{1}, x_{2}\right)
$$

for some $r \in[0,1)$.

Definition 2.4. ([5]) Let $(X, d)$ be a metric space and $M$ be a nonempty subset of $X . J: M \rightarrow 2^{X}$ is called a contractive type set-valued mapping if, for all $x \in M$ and $u_{x} \in J x$, there exists $v_{y} \in J y$ for all $y \in M$ such that

$$
d\left(u_{x}, v_{y}\right) \leq h d(x, y)
$$

for some $h \in[0,1)$.

Remark. It is obvious that a weakly $f$-contractive type set-valued mapping is more generalized than the weakly contractive type and contractive type set-valued mapppings.

Let $C(X)$ denote the family of all nonempty closed subsets of a metric space $(X, d)$ and $A$ be a set-valued mapping from $X$ into $C(X)$.

Definition 2.5. Let $p$ be a $\omega$-distance on $X$ and $f: X \rightarrow X$ be a mapping. A function $\phi: X \rightarrow[0,+\infty)$ is called a $(f, p)$-weak entropy of a set-valued mapping $A: X \rightarrow C(X)$ if, for all $x \in X$, there exists $y \in A x$ such that

$$
p(f x, f y) \leq \phi(x)-\phi(y)
$$

Definition 2.6. A set-valued mapping $A: X \rightarrow C(X)$ is said to be $(f, p)$-weakly dissipative if there exists a $(f, p)$-weak entropy of $A$.

Definition 2.7. A set-valued mapping $A: X \rightarrow C(X)$ is said to be upper semicontinuous if

$$
\lim _{n \rightarrow \infty} H_{+}\left(A x_{n}, A x\right)=0
$$

whenever $\left\{x_{n}\right\}$ is a sequence in $X$ such that $\lim _{n \rightarrow \infty} x_{n}=x \in X$, where

$$
H_{+}(S, T)=\sup _{y \in T x \in S} \inf d(x, y)
$$

for all $S, T \in C(X)$.

\section{Main results}

Now, we are ready to give our main theorems. 
Theorem 3.1. Let $(X, d)$ be a complete metric space, $M$ be a nonempty closed subset of $X$ and $p$ be a $\omega$-distance on $X$. Let $f: M \rightarrow M$ be a finction and $J: M \rightarrow 2^{M}$ be a weakly $f$-contractive type set-valued mapping with closed values. If $f(M)=M$, then there exists a point $z \in M$ such that $f z \in J z$.

Proof. Let $x_{0}$ be an arbitrary but fixed element of $M$ and choose $y_{1} \in J\left(x_{0}\right)$. Since $f(M)=M$, we can choose $x_{1} \in M$ such that $y_{1}=f x_{1}$. Moreover, since $J$ is weakly $f$-contractive type, there exists $y_{2} \in J x_{1}$ such that, for some $h \in[0,1)$,

$$
p\left(y_{1}, y_{2}\right) \leq h p\left(f x_{0}, f x_{1}\right) .
$$

Since $f(M)=M$, we can choose again $x_{2} \in M$ such that $f x_{2}=y_{2}$. Inductively, we can obtain a sequence $\left\{f x_{n}\right\}$ in $M=f(M)$ such that

$$
\left\{\begin{array}{l}
f x_{n+1} \in J x_{n} \\
p\left(f x_{n}, f x_{n+1}\right) \leq h p\left(f x_{n-1}, f x_{n}\right)
\end{array}\right.
$$

for $n=0,1,2, \ldots$. Thus we have

$$
\begin{aligned}
p\left(f x_{n}, f x_{n+1}\right) & \leq h p\left(f x_{n-1}, f x_{n}\right) \\
& \leq \cdots \\
& \leq h^{n} p\left(f x_{0}, f x_{1}\right)
\end{aligned}
$$

Since $h \in[0,1)$, it follows from (3.2) and Lemma 2.1 that $\left\{f x_{n}\right\}$ is a Cauchy sequence in $M=f(M)$. Hence, by the completeness of $M,\left\{f x_{n}\right\}$ converges to a point $u \in M$. Since $f(M)=M$, there exists $z \in M$ such that $f z=u$.

Furthermore, since $F x_{n+1} \in J x_{n}$ for $n=0,1,2, \ldots, J$ is weakly $f$-contractive type and $f(M)=M$, we can choose $f v_{n} \in J z$ such that

$$
p\left(f x_{n+1}, f v_{n}\right) \leq h p\left(f x_{n}, f z\right) .
$$

Since $f x_{n} \rightarrow f z=u$ as $n \rightarrow \infty$, by the lower semicontinuity of $p$, we have

$$
\begin{aligned}
p\left(f x_{n}, f z\right) & \leq \lim _{m \rightarrow \infty} p\left(f x_{n}, f x_{m}\right) \\
& \leq \lim _{m \rightarrow \infty} h^{m-n} p\left(f x_{0}, f x_{1}\right)
\end{aligned}
$$

for all $m, n \in N$ with $m>n$. Therefore, from (3.3), (3.4) and lemma 2.1, it follows that $f v_{n} \rightarrow f z$ as $n \rightarrow \infty$. Since $j z$ is closed, $f z \in J z$. This completes the proof.

Remark. If $f=I_{X}$ (: the identity mapping on $\left.X\right)$, then we obtain Theorem 2 in [6]. Further, if $f=I_{X}$ and $p=d$, then we have Theorem 2.3 in [2] as a corollary.

Next, we need the following lemma for a generalization of Caristi's fixed point theorem. The proof of the following lemma is similar to that of Lemma 1 in [6]: 
Lemma 3.2. Let $(X, d)$ be a metric space and $p$ be a $\omega$-distance on $X$. If $p\left(x_{n}, x_{m}\right) \leq$ $a_{n, m}$ for any $n, m \in N$ with $m>n$ and $a_{n, m} \geq 0$ with $a_{n, m} \rightarrow 0$ as $n, m \rightarrow \infty$, then $\left\{x_{n}\right\}$ is a Cauchy sequence in $X$.

Proof. Note that, for sufficiently large $m, n \in N$ with $m>n, a_{m, n} \rightarrow 0^{+}$. Let $\varepsilon \geq 0$. By the definition of $\omega$-distance, there exists $\delta>0$ such that $p(u, v) \leq \delta$ and $p(u, z) \leq \delta$ imply $d(v, z) \leq \varepsilon$. Thus, since $a_{m, n} \rightarrow 0^{+}$as $n, m \rightarrow \infty$, there exists a positive integer $n_{0} \in N$ such that $a_{n_{0}, m}<\delta$ for $n, m \geq n_{0}$, which implies that $p\left(x_{n_{0}}, x_{m}\right)<\delta$ and $p\left(x_{n_{0}}, x_{n}\right)<\delta$. Therefore, $d\left(x_{n}, x_{m}\right) \leq \varepsilon$, i.e., $\left\{x_{n}\right\}$ is a Cauchy sequence in $X$. This completes the proof.

Theorem 3.3. Let $(X, d)$ be a complete metric space and $p$ be a $\omega$-distance on $X$. If a function $f: X \rightarrow X$ is surjective and a set-valued mapping $A: X \rightarrow C(X)$ is $(f, p)$-weakly dissipative and upper semicontinuous, then there exists $z \in X$ such that $f z \in A z$ :

Proof. Let $\phi$ be a $(f, p)$-weak entropy of a set-valued mapping $A: X \rightarrow C(X)$. By (2.1), we can construct a sequence $\left\{x_{n}\right\}$ in $X$ such that

$$
\left\{\begin{array}{l}
f x_{n+1} \in A x_{n}, \\
p\left(f x_{n}, f x_{n+1}\right) \leq \phi\left(x_{n}\right)-\phi\left(x_{n+1}\right)
\end{array}\right.
$$

for $n=0,1,2, \ldots$. From (3.5), it follows that $\phi\left(x_{n}\right) \geq 0$ for $n=0,1,2, \ldots$ and $\left\{\phi\left(x_{n}\right)\right\}$ is nonincreasing.

Suppose that $\phi\left(x_{n}\right) \rightarrow a \in[0, \infty)$ as $n \rightarrow \infty$. Then, since, for any $i, j \in N$ with $i<j$, we have

$$
\begin{aligned}
p\left(f x_{i}, f x_{j}\right) & \leq \sum_{n=i}^{j-1} p\left(f x_{n}, f x_{n+1}\right) \\
& \leq \sum_{n=i}^{j-1}\left(\phi\left(x_{n}\right)-\phi\left(x_{n+1}\right)\right) \\
& =\phi\left(x_{i}\right)-\phi\left(x_{j}\right),
\end{aligned}
$$

from Lemma 3.2, it follows that $\left\{f x_{n}\right\}$ is a Cauchy sequence in $X$. Since $(X, d)$ is complete, $\left\{f x_{n}\right\}$ converges to a point $u \in X$. since $f$ is surjective, there exists $z \in X$ such that $f z=u$. Furthermore, since $f x_{n+1} \in A x_{n}$, we have

$$
d\left(f x_{n+1}, A z\right) \leq H_{+}\left(A z, A x_{n}\right) .
$$

Thus, since $A$ is upper semicontinuous, $\lim _{n \rightarrow \infty} H_{+}\left(A z, A x_{n}\right)=0$. On the other hand, we have

$$
\lim _{n \rightarrow \infty} d\left(f x_{n+1}, A z\right)=d(f z, A z) .
$$

Therefore, from (3.7), it follows that $d(f z, A z)=0$ and so, since $A z$ is closed, $f z \in A z$. This completes the proof. 
Remark. From Theorem 3.3, we have Caristi's fixed point theorem [1] as a corollary.

The following example illustrates our theorem:

Example. Let $X=\{1,2,3,4\}$ and define $d, p: X \times X \rightarrow[0, \infty)$ as follows, respectively:

$$
\begin{aligned}
& d(1,1)=d(2,2)=d(3,3)=d(4,4)=0, \\
& d(1,2)=d(2,1)=1, d(1,3)=d(3,1)=2, \\
& d(1,4)=d(4,1)=3, d(2,3)=d(3,2)=2, \\
& d(1,1)=d(4,2)=3, d(3,4)=d(4,3)=1
\end{aligned}
$$

and

$$
\begin{aligned}
& p(1,1)=1, p(1,2)=1, p(1,3)=2, p(1,4)=3, \\
& p(2,1)=2, p(2,2)=1, p(2,3)=3, p(2,4)=2, \\
& p(3,1)=2, p(3,2)=1, p(3,3)=2, p(3,4)=1 \\
& p(4,1)=1, p(4,2)=2, p(4,3)=2, p(4,4)=2 .
\end{aligned}
$$

Clearly $d$ is a metric and $p$ is a $\omega$-distance on $X$, respectively. Define $f: X \rightarrow X$ by

$$
f(1)=2, f(2)=3, f(3)=1, f(4)=4
$$

and $\phi: X \rightarrow[0,+\infty)$ by

$$
\phi(1)=8, \phi(2)=4, \phi(3)=7, \phi(4)=6 .
$$

Next, define $A: X \rightarrow C(X)$ by

$$
A(1)=\{2,3\}, A(2)=\{3,4\}, A(3)=\{1,2\}, A(4)=\{2,4\} .
$$

By routine computation, it is easy to check that all the conditions of Theorem 3.3 are satisfied. Moreover, for all $z \in X$, we have $f z \in A z$.

\section{Acknowledgement}

The authors would like to express their deep thanks to the referee for his helpful suggestions.

\section{References}

[1] J. Caristi, Fixed Point theorems for mappings satisfying inwardness conditions, Trans. Amer. Math. Soc. 215(1976), 241-251.

[2] S. S. Chang, N. J. Huang and Y. J. Cho, Coincidence and fixed point theorems, Bull. Honam Math. Soc. 12(1995), 153-161. 
[3] T. Husain and E. Tarafdar, Fixed point theorems for multivalued mappings of nonexpansive type, Yokohama Math. J. 28 (1980), 1-6.

[4] T. Husain and A. Latif, Fixed points of multivalued nonexpansive maps, Math. Japon. 33(1988), 285-391.

[5] T. Husain and A. Latif, Fixed points of multivalued maps, Internat. J. Math. and Math. Sci. 14 (1991), 421-430.

[6] O. Kada, T. Suzuki and W. Takahashi, Nonconvex minimization theorems and fixed point theorems in complete metric spaces, Math. Japon. 44(1996), 381-391.

[7] S. B. Nadler, Jr., Multivalued contraction mappings, Pacific J. Math. 30(1969), 475-488.

[8] T. Suzuki and W. Takahashi, Fixed point theorems and characterizations of metric completeness, Topological Methods in Nonlinear Analysis 8(1996), 371-382.

[9] H. K. Xu, On weakly nonexpansive and *-nonexpansive multivalued mappings, Math. Japon. $36(1991), 441-445$.

Department of Mathematics, Gyeongsang National University, Chinju 660-701, Korea

Department of Mathematics, Sichuan University, Chengdu, Sichuan 610064, People's Republic of China 